\title{
The Change of Bone Mineral Density by Bisphosphonates Therapy with Calcium-Antagonists in Osteoporosis
}

\author{
Soon-Joo Kim ${ }^{1}$, Hyen-Oh La ${ }^{2}$ and Young-Sook Kang ${ }^{1 *}$ \\ ${ }^{1}$ College of Pharmacy and Research Institute of Pharmaceutical Science, Sookmyung Women's University, Seoul, Korea \\ ${ }^{2}$ Pharmacology, College of Medicine, The Catholic University, Seoul, Korea
}

(Received June 12, 2008; Accepted June 19, 2008)

\begin{abstract}
Imbalance in calcium and phosphorous metabolism due to aging or menopause leads to osteoporosis. In contrast to patients with normal blood pressure, hypertensive patients have a higher loss of calcium in the urine with its attendant risk of osteoporosis. The high blood pressure is associated with the risk of bone loss and abnormalities in calcium metabolism leading to calcium loss. So we retrospectively investigated the changes of bone mineral density (BMD) which drugs can have clinical influences over osteoporosis treatments of patients with calcium-antagonists as common antihypertensive drugs and with bisphosphonates which causes a most effective inhibition of osteoclasts resorption. As a result over 70 years of age group and within bisphosphonates group, alendronate $70 \mathrm{mg}$ once-weekly group showed significant increase of BMD in lumbar area. Combination group of cilnidipine and maxmarvil ${ }^{\circledR}$ showed very significant decrease of BMD. In conclusion, it is desirable that combination therapy with calcium-antagonists is used carefully in the treatment of osteoporosis with high blood pressure.
\end{abstract}

Keywords: bone mineral density, bisphosphonates, alendronate, calcium-antagonists, cilnidipine

\section{INTRODUCTION}

The high blood pressure among elderly women is statistically associated with decreased bone mineral content at the femoral neck, which may increase the susceptibility to fractures (Cappuccio et al., 1999). In contrast to patients with normal blood pressure, hypertensive patients have a higher loss of calcium in the urine with its attendant risk of osteoporosis. The high blood pressure is associated with the risk of bone loss and abnormalities in calcium metabolism leading to calcium loss (Cappuccio et al., 1999).

The bone mineral density (BMD) is used for diagnosis of osteoporosis and provides an index of whole calcium balance. It implies that elevated blood pressure is inversely correlated with reduced BMD in with essential hypertension, indicating that high blood pressure is associated with abnormalities in calcium metabolism and insulin resistance (Gotoh et al., 2005).

The aim of osteoporosis therapy is to increase bone mass and prevent fractures, leading to morbidity and mortality decrease. It includes 'treatment of pain, initia-

\footnotetext{
${ }^{*}$ Corresponding author

Tel: +82-2-710-9562, Fax: +82-2-2077-7975

E-mail: yskang@sookmyung.ac.kr
}

tion of physical activity and exercises, prevention of fractures, adaptation of life-style for skeletal health, boneconscious nutrition, vitamin $\mathrm{D}$ and calcium supplements, hormone replacement therapy (HRT) for short periods only, antiresorptive therapy (bisphosphonates, raloxifene, calcitonin), osteoanabolic therapy (fluoride, strontium, anabolics, parathormone), and other medications (statins, growth factors, tetracyclins, leptin)' (Caren 2002, Reiner and Bertha 2003, Shun-ichi 2003).

Bisphosphonates, especially nitrogen-containing bisphosphonates, have a high affinity for certain structures on the surface of bone. Most of the bisphosphonates absorbed in the gastrointestinal tract is deposited on the bone within hours, especially in the acidic environment under the osteoclasts. This causes the most effective inhibition of osteoclasts resorption, as well as reactivation of the suppressed osteoblasts and thereby an overall positive bone-balance and increase in bone mass. In the long term, inhibition of resorption results in a positive calcium balance with a continuous increase in bone mass (Reiner and Bertha 2003). So we retrospectively investigated the changes of BMD which drugs can have clinical influences over osteoporosis treatments of patients with calcium-antagonists among antihypertensive drugs with bisphosphonates. 


\section{METHODS}

We did complete enumeration over total 868 outpatients to whom osteoporosis therapy were prescribed in a general hospital from Jan, 2004 to Dec, 2006.

Method was retrospective investigation of patient medical records. We investigated kinds of used drugs for total 868 outpatients, and the medical departments where patients were prescribed osteoporosis therapy and diagnosis. We focused on the kinds and frequencies of osteoporosis drugs and daily dose of calcium-antagonists among antihypertensive drugs, which were used for 90 patients taking calcium-antagonists among 120 patients taking antihypertensive drugs (Table I).

Also, we did investigational comparison for BMD to find out the influence of combination of osteoporosis therapy and calcium-antagonists to 49 patients taking calciumantagonists among antihypertensive drugs at least for 6 months with confirmed change on more than two BMD tests.

BMD data obtained through quantitative BMD measurement of DXA (dual energy X-ray absorptiometry) was comparatively analyzed. LBMD (BMD of lumbar spine) and WBMD (BMD of Ward's) were used for data analysis. Initial observation value (the first observation value) and final value (the last observation value regardless of measurement frequencies) was used for measuring BMD. All data were represented by mean \pm SEM. Statisti-

Table I. Drugs in combination therapy

\begin{tabular}{cl}
\hline Efficacy & \multicolumn{1}{c}{ General name } \\
\hline & Alendronate (ALD) \\
& Risedronate (RSD) \\
& Pamidronate (PAMD) \\
Osteoporosis drugs & Maxmarvil ${ }^{\circledR}$ (MXM) \\
& Raloxifene (RAXF) \\
& Menatetrenone (MENAT) \\
& Salcatonine (R-SLT) \\
& Amlodipine (AMDP) \\
& Lacidipine (LADP) \\
& Lercanidipine (LCDP) \\
& Nifedipine (NFP) \\
& Cilnidipine (CNDP) \\
Calcium-antagonists & Diltiazeme (DTZ) \\
& Verapamil (VPM) \\
\hline
\end{tabular}

We investigated kinds of used drugs in the combination drugs, osteoporosis drugs with antihypertensive drugs. cal comparison between groups and treatments was performed using student's $t$-test.

\section{RESULTS}

\section{Analysis of patients taking osteoporosis drugs}

(1) General characteristics of osteoporosis drugs medication group

Among 868 patients taking osteoporosis drugs, 78 patients $(9.0 \%)$ were male and 790 patients $(91 \%)$ were female. Mean age was $61.2 \pm 14.7$ years. Age grouped distribution was 170 patients under 49 years, 414 patients (48\%) between 50 and 69 years of age and 284 patients over 70 years of age.

Analysis of medical department where osteoporosis drugs were prescribed showed even distribution including gynecology (GY) 220 cases (25\%), orthopedic surgery (OS) 206 cases (24\%), and medical endocrinology (ME) 111 cases $(13 \%)$.

(2) Analysis of diagnosis (Repetition selection)

Diagnosis of 868 patients yielded 1,561 cases of osteoporosis to base our studies. Among them 392 cases $(25 \%)$ state 'osteoporosis' followed by related skeletal and articular diseases in 148 cases (9.5\%), back pain in 76 cases $(4.9 \%)$ and fracture in 69 cases $(4.4 \%)$. Apart from these, chronic diseases were such as diabetes, gastrointestinal and endocrinological diseases evenly distributed.

\section{Analysis of combination therapy group of bispho- sphonates and calcium-antagonists}

Patients with calcium-antagonists therapy of total 120 patients with combination therapy were 90 persons, 12 male patients (13\%) and 78 female patients $(87 \%)$.

\section{(1) Frequencies of the bisphosphonates}

Analysis of prescribed the bisphosphonates in the combination therapy group with antihypertensive drugs $(\mathrm{n}=90)$ shows that bisphosphonate drugs (72 cases, $80 \%$ ) was the most commonly prescribed drug and among them alendronate (ALD) was 33 cases $(37 \%)$, risedronate (RSD) 27 cases $(30 \%)$, maxmarvil ${ }^{\circledR}(\mathrm{MXM})$ of complex for ALD and calcitriol 16 cases (18\%).

(2) Frequencies and dose analysis of calcium-antagonists medication $(n=90)$

Amlodipine (32 cases, 36\%) was the most commonly used calcium-antagonists followed by nifedipine and cilnidipine (22 cases, 24\%), lacidipine (8 cases, $8.9 \%$ ). cal- 
cium-antagonists dose of all the 90 cases was within reference range and it was medicated once or twice a day.

\section{BMD analysis of calcium-antagonists medication group}

Patients with change confirmation during more than two BMD tests were 49 persons (51\%). Among them two persons $(4.1 \%)$ were male and 47 persons were female (96\%). Age grouped distribution were 14 persons under 49 years, 16 persons between 50 and 69 years and 19 persons over 70 years.

(2) Analysis grouped by the bisphosphonates ( $n=49$ )

Medication frequencies of bisphosphonates were ordered by 20 cases $(41 \%)$ with ALD, 15 cases $(31 \%)$ with risedronate (RSD) and 9 cases (18\%) with maxmar$\mathrm{vil}^{\circledR}$ (MXM).

(3) Comparison analysis of BMD change within calcium-antagonists medication group

(1) Comparison of change between initial and final BMD $(n=49)$

Hypothesis testing about change between initial BMD and final BMD within calcium-antagonists medication group showed significant LBMD increase $(p=0.0017)$ (Fig. 1).

(2) Comparison between initial and final BMD by age $(n=49)$

Age group of over 70 years showed significant increase in LBMD ( $p=0.0036$ ) (Fig. 2).

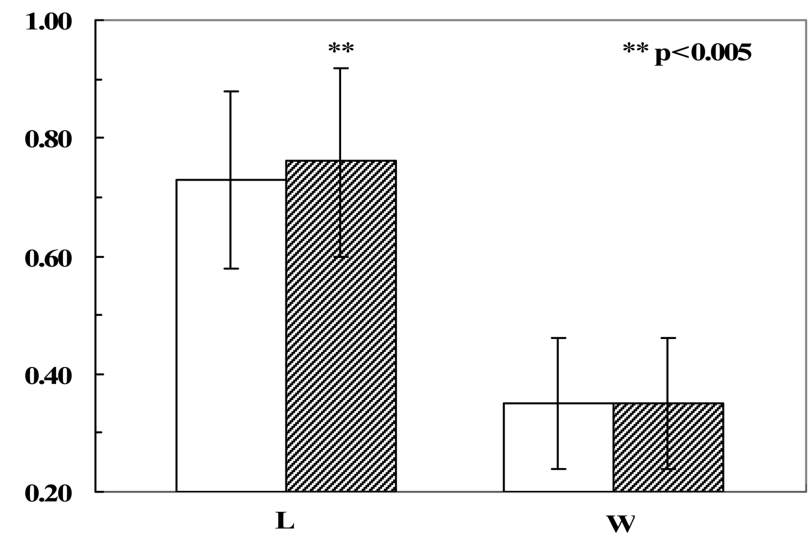

Fig. 1. Comparison of change between initial and final BMD within calcium-antagonists medication group showed significant LBMD increase $(n=49)$.

The area of lumbar and Ward's of femur is presented $L$ and W, respectively.

Initial $\mathrm{BMD}(\square)$, final $\mathrm{BMD}(\square \Delta) .{ }^{* \star} p<0.005$, significantly different from initial BMD.

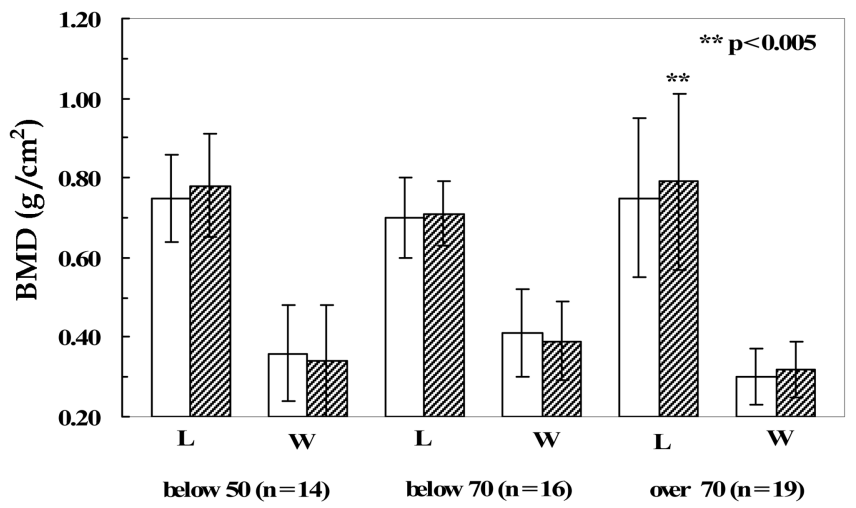

Fig. 2. Comparison between initial and final BMD by age within calcium-antagonists medication group $(n=49)$; Over 70 years of age group showed significant increase in LBMD. ${ }^{* *} p<0.005$, significantly different from initial BMD.

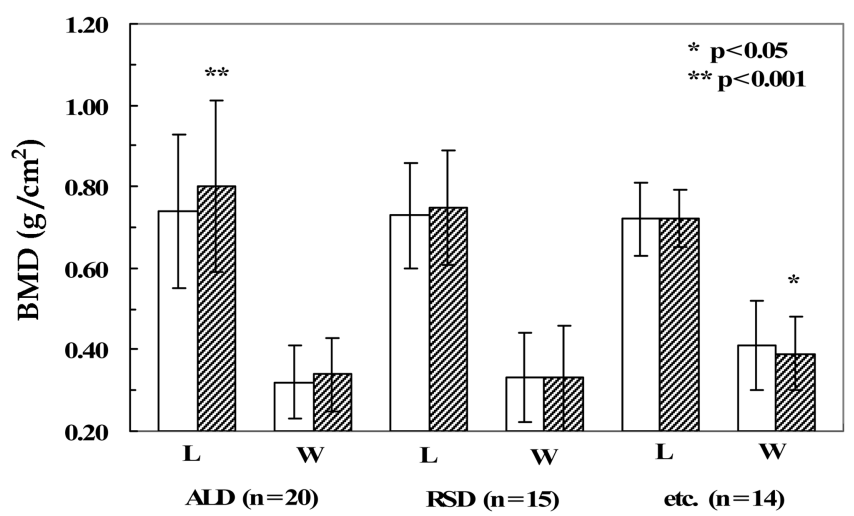

Fig. 3. Analysis grouped by osteoporosis drugs within calciumantagonists medication group ( $n=49)$; ALD group showed significant increase in LBMD and other groups shows significant decrease in Ward's area of femur (WBMD) in the analysis grouped by osteoporosis drugs.

${ }^{*} p<0.05,{ }^{* *} p<0.001$, significantly different from initial BMD.

(3) Analysis grouped by the bisphosphonates $(n=49)$

ALD group $(n=20)$ showed significant increase in LBMD $(p=0.0004)$ and other groups showed significant decrease in WBMD $(p=0.0393)$ in the analysis grouped by bisphosphonates (Fig. 3).

(4) Comparison of BMD change in the bisphosphonates and calcium-antagonists combination $(n=41)$

Analysis bisphosphonates dose shows that ALD 70mg once-weekly group is the most as 18 cases. Combination groups with more than two cases only were analyzed. BMD change is as follows. Combination of CNDP and MXM shows very significant decrease in WBMD ( $p=$ 0.0089), and combination of AMDP, ALD in LBMD and combination of MXM in WBMD showed significant increase $(p=0.0385,0.0363)$ and also combination of 
Table II. Comparison of BMD change in osteoporosis drugs and calcium-antagonists combination

\begin{tabular}{|c|c|c|c|c|c|}
\hline \multirow{2}{*}{$\begin{array}{c}\text { Osteoporosis } \\
\text { drugs }\end{array}$} & \multirow{2}{*}{$\begin{array}{l}\text { Calcium } \\
\text { antagonist }\end{array}$} & \multirow{2}{*}{ Sites } & \multicolumn{2}{|c|}{$\operatorname{BMD}\left(\mathrm{g} / \mathrm{cm}^{2}\right)$} & \\
\hline & & & Initial & Final & \\
\hline \multirow{6}{*}{ ALD } & \multirow{2}{*}{ AMDP } & $L$ & $0.72 \pm 0.12$ & $0.77 \pm 0.14^{*}$ & \multirow{2}{*}{$(n=7)$} \\
\hline & & w & $0.34 \pm 0.06$ & $0.35 \pm 0.04$ & \\
\hline & \multirow{2}{*}{ NFP } & L & $0.66 \pm 0.08$ & $0.71 \pm 0.09$ & \multirow{2}{*}{$(n=8)$} \\
\hline & & W & $0.30 \pm 0.11$ & $0.33 \pm 0.14$ & \\
\hline & \multirow{2}{*}{ LADP } & L & $0.94 \pm 0.34$ & $1.03 \pm 0.37^{\star}$ & \multirow{2}{*}{$(n=4)$} \\
\hline & & W & $0.30 \pm 0.10$ & $0.31 \pm 0.07$ & \\
\hline \multirow{6}{*}{ RSD } & \multirow{2}{*}{ AMDP } & $L$ & $0.78 \pm 0.17$ & $0.83 \pm 0.21$ & \multirow{2}{*}{$(n=4)$} \\
\hline & & W & $0.37 \pm 0.21$ & $0.36 \pm 0.23$ & \\
\hline & \multirow{2}{*}{ NFP } & $\mathrm{L}$ & $0.73 \pm 0.08$ & $0.76 \pm 0.08$ & \multirow{2}{*}{$(n=5)$} \\
\hline & & w & $0.33 \pm 0.06$ & $0.33 \pm 0.10$ & \\
\hline & \multirow{2}{*}{ CNDP } & L & $0.71 \pm 0.18$ & $0.69 \pm 0.16$ & \multirow{2}{*}{$(n=5)$} \\
\hline & & W & $0.29 \pm 0.06$ & $0.27 \pm 0.07$ & \\
\hline \multirow{6}{*}{ MXM } & \multirow{2}{*}{ AMDP } & $L$ & $0.71 \pm 0.02$ & $0.69 \pm 0.01$ & \multirow{2}{*}{$(n=2)$} \\
\hline & & W & $0.32 \pm 0.07$ & $0.36 \pm 0.07^{\star}$ & \\
\hline & \multirow{2}{*}{ LADP } & L & $0.70 \pm 0.04$ & $0.67 \pm 0.06$ & \multirow{2}{*}{$(n=2)$} \\
\hline & & W & $0.41 \pm 0.07$ & $0.39 \pm 0.06$ & \\
\hline & \multirow{2}{*}{ CNDP } & L & $0.83 \pm 0.08$ & $0.80 \pm 0.05$ & \multirow{2}{*}{$(n=4)$} \\
\hline & & W & $0.52 \pm 0.09$ & $0.45 \pm 0.09^{* *}$ & \\
\hline
\end{tabular}

Combination groups with more than two cases only were analyzed. Bone mineral density (BMD) change is that combination of CNDP and MXM shows very significant decrease in WBMD. The BMD of lumbar and Ward's of femur is represented L and W, respectively.

${ }^{\star} p<0.05 ;{ }^{* *} p<0.01$, significantly different from initial BMD.

LADP and ALD showed significant increase in LBMD ( $p$ $=0.0119$ ) (Table II).

\section{DISCUSSION}

During this study, patients with osteoporosis medication between 50 and 69 years of age were the most common age group. These are consistent with the increase of osteoporosis prevalence following hormone excretion decrease in the $50 \mathrm{~s}$, due to menopausal age (Kim et al., 1988).

Distribution over medical department where osteoporosis drugs were prescribed shows high ratio for gynecology (GY) and orthopedic surgery (OS). Those are consistent with the facts that in case of GY, $91 \%$ of patients were female, mean age was 61 years, menopause made change of serum lipid metabolism and increased risk factor for osteoporosis, and in case of OS, aging causes degeneration of articulation. Diagnosis distribution showed that patients had not only skeletal disease and fracture but also more than two chronic diseases such as diabe- tes mellitus or hypertension. Drugs for that also showed various pattern.

In comparing the initial and final bone mineral density (BMD) value in the osteoporosis drugs group only alendronate (ALD) medication group showed significant increase in LBMD (BMD of lumbar spine). ALD $70 \mathrm{mg}$ onceweekly showed significantly greater increases in lumbar spine and hip BMD and greater reductions in markers of bone turnover than raloxifen $60 \mathrm{mg}$ daily over 12 months in postmenopausal osteoporosis (Luckey et al., 2004, Sambrook et al., 2004). ALD $70 \mathrm{mg}$ once-weekly yielded significantly greater BMD gains, in total hip BMD and lumbar spine BMD, and larger decreases in bone turnover marker levels than risedronate $35 \mathrm{mg}$ once-weekly over 24 months, with no difference in upper gastrointestinal tolerability (Rosen et al., 2005, Reid et al., 2008). Comparative study of combination therapy group of osteoporosis drugs and calcium-antagonists was as follows. The amlodipine group and lacidipine group combined with ALD $70 \mathrm{mg}$ once-weekly showed significant increase in LBMD. Amlodipine combined with maxmar- 
$\mathrm{vil}^{\circledR}$ group showed significant increase in WBMD (BMD of Ward's), but cilnidipine group showed significant decrease in WBMD. Theses results are similar with those of ALD dose as following results.

The treatment with $1,2.5$, or $5 \mathrm{mg}$ over 2 years, and $10 \mathrm{mg}$ of ALD daily for 3 years increased significantly dose-related increases in the lumbar spine BMD $(0.65 \%$, $3.5 \%, 5.7 \%$, and $8.8 \%$ respectively). Also, ALD produced dose-dependent decreases in bone turnover and increases in mineral density in the lumbar spine, and skeleton and reduces the incidence of fractures in postmenopausal women with osteoporosis (Bone et al., 1997, Liberman et al., 1995). Treatment with $5,10 \mathrm{mg}$ of ALD daily for 10 years, or the discontinuation of $20 \mathrm{mg}$ of ALD daily for 2 years, the $10 \mathrm{mg}$ group increased the BMD of $14 \%$ in lumbar spine. But smaller gains occurred in $5 \mathrm{mg}$ group, and the discontinuation group resulted in the gradual loss of effect. This was associated with sustained therapeutic effects on bone density and remodeling (Bone et al., 2004). Ten mg once-daily dosing is effective for increasing BMD and ALD $70 \mathrm{mg}$ once-weekly dosing regimen provide patients with a more convenient, therapeutically equivalent alternative to $10 \mathrm{mg}$ daily dosing (Schnitzer et al., 2000). In the experiment of rat osteocyte, high dose $(100 \mathrm{mg} / \mathrm{kg})$ nifedipine or verapamil of calcium channel blockers (CCBs) inhibit load-induced bone formation by $\mathrm{Ca}^{++}$blocking effect on BMD (Jiliang et al., 2002), but verapamil within clinical reference range $(0.75 \mathrm{mg} / \mathrm{kg})$ shows increase BMD and bone formation (Eva et al., 2001). Nifedipine leads to a lower bone formation and epiphyseal growth plate activity, and also was affected bone physiology with consequences on bone growth and bone volume when compared with an untreated control group (Duriez et al., 1993). Chronic nifedipine has not been shown to affect bone mineral content and BMD (Albers et al., 1991).

CCBs are the most used drugs to treat mild-to-moderate hypertension and especially amlodipine of slow-onset, long-acting CCBs is commonly used for antihypertensive action and few adverse effects (Takao, 1998). Amlodipine is the most used drug in this study. Benidipine blocks calcium influx through L-type voltage dependent calcium channel greatly potentiate mineralization, calcium influx and alkaline phosphates (ALP) activity. Amlodipine partially elevate ALP activity and block the calcium influx at $10 \mu \mathrm{M}$ in osteobalst-like cell (MC3T3-E1 cell) line (Nishiya and Sugimoto, 2001). Also benidipine blocks calcium influx more strongly than other CCBs by high affinity to calcium channel and has strong osteoblast differentiating ability. Other dihydropyridine derived CCBs also stimulate osteoblast differentiation (Nishiya et al., 2002).

CCBs receptors linked to voltage-regulate calcium channels have been reported in osteoblast-like osteosarcoma cell culture lines (Guggino et al., 1988). The properties of calcium channels are largely conferred by their pore-forming $\alpha_{1}$ subunit. And the dihydropyridine (DHP) is bound with high affinity the $\alpha_{1}$ subunit of the DHP receptor protein (Mori et al., 1996). Barry was reported that calcium channel $\alpha_{1}$ subunits are expressed in human osteoblasts including both L-type and non L-type isoforms (Barry, 2000). Above facts suggest that L-type calcium channel plays an important role in ability for osteoblast and bone metabolism. So osteocyte reaction of CCBs related with L-type calcium channel is important.

It was reported, in an ovariectomy induced osteopenic rat model, that amlodipine was found to both promote femoral calcium deposition and inhibit its loss, but lacidipine was found only to inhibit calcium loss. The potent action of amlodipine and lacidipine compared to other drugs used to treat osteoporosis, and may lead to finding a novel therapeutic target for bone diseases such as osteoporosis, at least in hypertensive patients (Zekai et al., 2008). But because there is no report about bone formation or bone metabolism of cilnidipine, in the case of combination therapy of cilnidipine and risedronate or maxmarvil ${ }^{\circledR}, \mathrm{BMD}$ decrease in lumbar and femur area can not be compared. So we assume more in vitro experiment of osteocytes over all the dihydropyridine drugs including cilnidipine and more clinically prospective in vivo study should be done. Cilnidipine combination therapy group shows decreased trend of BMD but no statistical significance in nonparametric test (Wilcoxon singed rank test). So, more patients with calcium-antagonist combination therapy are needed in next study.

In conclusion, it is desirable that combination therapy with calcium-antagonists is used carefully in the treatment of osteoporosis with high blood pressure. In the case of combination therapy of osteoporosis drugs and above, BMD change between intervals need to be prospectively studied and when developing new calciumantagonists clinical trial result related to various disease as well as in vitro result should be considered and applied to clinical conditions.

\section{REFERENCES}

Albers, M.M., Johnson, W.V., and Jackson, R.D. (1991). Chronic use of the calcium channel blocker nifedipine has no significant effect on bone metabolism in men. Bone. 12, 39-42.

Barry, E.L.R. (2000). Expression of mRNAs for the $\alpha_{1}$ subunit of 
voltage-gated calcium channels in human osteoblast-like cell lines and in normal human osteobasts. Calcif Tissue Int. 66, 145-150.

Bone, H.G., Robert, W.D., Joseph, R.T., Steven, T.H., Robert, S.W., Angelo, A.L., Michael, R.M., Donald, B.K., Barry, J.G., Erika, H., and William J.P. (1997). Dose-response relationships for alendronate treatment in osteoporotic elderly women. J Clini Endocrinol Metab. 82(1), 265-274.

Bone, H.G., Hosking, D., Devogelaer, J.P., Tucci, J.R., Emkey, R.D., Tonino, R.P., Rodrigue-Portales, J.A., Downs, R.W., Gupta, J., Santora, A.C., and Liberman U.A. (2004). Ten years' experience with alendronate for osteoporosis in postmenopausal women. N Engl Med., 350, 1189-1899.

Cappuccio, F.P., Meilahn, E., Zmuda, J.M., and Cauley, J.A. (1999). High blood pressure and bone-mineral loss in elderly white women: a prospective study. Study of osteoporotic fractures research group. Lancet. 354, 971-975.

Caren, G.S. (2002). Bisphosphonates and Osteoporosis., N Eng J Med. 346(9) 642.

Duriez, J., Flautre, B., Blary, C., and Hardouin, P. (1993). Effects of the calcium channel blocker nifedipine on epiphyseal growth plate and bone turnover: a study in rabbit. Calcif Tissue Int. 52, 120-124

Eva, S., Diane, M.C., Mohammed, P.A., and Donald, B.K. (2001). No effect of verapamil on the local bone response to in vivo mechanical loading. J Orth R. 19, 328-336.

Gotoh, M., Mizuno, K., Ono, Y., and Takahashi, M. (2005). High blood pressure, bone-mineral loss and insulin resistance in women. Hypertens Res. 28(7), 565-570.

Guggino, S.E., Wagner, J.A., Snowman, A.M., Hester, L.D., Sacktor, N., and Snyder, S.H. (1988). Phenylalkylamine-sensitive calcium channels in osteoblast-like osteosarcoma cells. $J$ Biol Chem, 263, 10155-61.

Jiliang, L.I., Randall, L.D., David, B.B., and Charles, H.T. (2002). L-type calcium channels mediate mechanically induced bone formation in vivo. J Bone Mine. 17(10), 1795-1800.

Kim, B.C., Kim, D.H., and Hur, M. (1988). A study of symptomatology, lipid change and hormonal change in the menopausal women. Korean J Obstet Gynecol. 31(6), 784-794.

Liberman, U.A., Weiss, S.R., Broll, J., Minne, H.W., Quan, H., Bell, N.H., Rodriguez-Portales, J., Downs, R.W. Jr., Dequeker, J., and Favus, M. (1995). Effect of oral alendronate on BMD and the incidence of fractures in postmenopausal osteoporosis. N Engl Med., 333(22), 1437-1443.

Luckey, M., Kagan, R., Greenspan, S., Bone, H., Kiel, R.D., Simon, J., Sackarowitz, J., Palmisano, J., Chen, E., Petrus- chke, R.A., and de Papp, A.E. (2004). Once-weekly alendronate $70 \mathrm{mg}$ and raloxifene $60 \mathrm{mg}$ daily in the treatment of postmenopausal osteoporosis. Menopause. 11(4), 405-415.

Mori, Y., Mikala, G., Vardi, G., Kobayashi, T., Koch, S., Wakamori, M., and Schwartz A. (1996). Molecular pharmacology of voltage-dependent calcium channels. Jpn J Pharmacol. 72 , 83-109.

Nishiya, Y. and Sugimoto, S. (2001). Effects of various antihypertensive drugs on the function of osteoblast. Biol. Pharm. Bull. 24(6), 628-633.

Nishiya, Y., Kosaka, N., Uchii, M., and Suginoto, S. (2002). A potent 1,4-dihydropyridine L-type calcium channel blocker, benidipine, promotes osteoblast differentiation. Calcif Tissue Int. 70, 30-39.

Reid, D.M., Hosking, D., Kendler, D., Brandi, M.L., Wark, J.D., Marques-Neto, J.F., Weryha, G., Vebruggen, N., Hutad, C.M., Mahlis, E.M., and Melton, M.E. (2008). A comparison of the effect of alendronate and risedronate on bone mineral density in postmenopausal women with osteoporosis: 24 month results from FACTS-International. Int J Clin Pract. 62(4), 575584.

Reiner, B. and Bertha, F. (2003). Osteoporosis. Springer.

Rosen, C.J., Hochberg, M.C., Bonnick, S.L., McClung, M., Miller, P., Broy, S., Kagan, R., Chen, E., Sambrook, P.N., Geusens, P., Ribot, C., Solimano, J.A., Ferrer-Barriendos, J., Gaines, K., Verbruggen, N., and Melton M.E. (2004). Alendronate produces greater effects than raloxifen on bone density and bone turnover in postmenopausal women with low bone density: results EFFECT International, $J$ Int Medicine. 255, 503-511.

Schnitzer, T., Bone, H.G., Crepaldi, G., Adami, S., McClung, M., Kiel, D., Felsenberg, D., Recker, R.R., Tonino, R.P., Roux, C., Pinchera, A., Foldes, A.J., Greenspan, S.L., Levine, M.A., Emkey, R., Santora, A.C. 2nd., Kaur, A., Thompson, D.E., Yates, J., and Orloff, J.J. (2000), Therapeutic equivalence of alendronate $70 \mathrm{mg}$ once-weekly and alendronate $10 \mathrm{mg}$ daily in the treatment of osteoporosis., Aging(Milano). 12(1), 1-12.

Shun-ichi, H. and Gideon, A.R. (2003). Control of osteoblast function and regulation of bone mass., Nature. 423, 349-355.

Takao, S. (1998). Current status of calcium-antagonists in Japan. Am J Cardio., 82(9B), 32R-34R.

Zekai, H., Bunyamin, B., Yuksel, O., Elif, C., and Halis, S. (2008). Protective effects of amlodipine and lacidipine on ovariectomy-induced bone loss in rats. Eur $J$ Pharmaco, 579, 241-245. 